\title{
Disorder effects in the anomalous Hall effect induced by Berry curvature
}

\author{
N. A. Sinitsyn, ${ }^{1}$ Qian Niu, ${ }^{1}$ Jairo Sinova, ${ }^{2}$ and Kentaro Nomura ${ }^{1}$ \\ ${ }^{1}$ Department of Physics, University of Texas at Austin, Austin Texas 78712-1081, USA \\ ${ }^{2}$ Department of Physics, Texas A\&M University, College Station, Texas 77843-4242, USA
}

(Received 25 February 2005; published 20 July 2005)

\begin{abstract}
We describe the charge transport in ferromagnets with spin-orbit coupled Bloch bands by combining the wave-packet evolution equations with the classical Bolzmann equation. This approach can be justified in the limit of smooth disorder potential. Besides the skew scattering contribution, we demonstrate how other effects of disorder appear which are closely linked to the Berry curvature of the Bloch states associated with the wave packet. We show that, although being of the same order of magnitude as the clean limit contribution, generally disorder corrections depend differently on various parameters and can lead to the sign reversal of the Hall current as the function of the chemical potential in systems with a nonconstant Berry curvature in momentum space. Earlier conclusions on the effects of disorder on the anomalous Hall effect depended stricly on the lack of momentum dependence of the Berry curvature in the models studied and generalizations of their findings to other systems with more complicated band structures were unjustified.
\end{abstract}

DOI: $10.1103 /$ PhysRevB.72.045346

PACS number(s): 73.20.Fz, 72.10.Fk, 72.15.Rn

\section{INTRODUCTION}

The theory of the anomalous Hall effect (AHE) has a long history. The appearance of the Hall current requires breaking of some basic symmetries. It was proposed by Karplus and Luttinger in the early 1950s (Ref. 1) that the anomalous Hall effect in ferromagnets results from the interplay of the exchange field, which breaks the time-reversal symmetry, and the spin-orbit coupling, that violates the chiral symmetry. Interestingly, at the same time a similar effect was predicted and explained in geometrical optics, ${ }^{2}$ however, its relation to the AHE was revealed only recently ${ }^{3,4}$ after the modern interpretation of both effects in terms of the Berry phase, which affects the motion of wave packets, had been constructed..$^{5-7}$ Luttinger $^{8}$ built a detailed theory of the AHE based on the high-order quantum Boltzmann equation calculations. ${ }^{9}$ In that work Luttinger identified various contributions, known today as Berry phase contribution, the skew scattering, the side jump on the impurity potential, and a contribution that involves interference from many scatterers.

Since then, a number of theoretical works appeared that extended the theory. However, until recent time most of them had been devoted to what is today called the extrinsic AHE. In the extrinsic AHE a simple Bloch band structure of the system is assumed and the spin-orbit interaction is localized on the impurity potential via terms of the form $\lambda_{S O} \hat{\sigma}_{z} \hat{\mathbf{z}} \mathbf{k}$ $\times \nabla V_{\text {dis }}(x)$, etc. It was recognized by Smit, Berger, and others $^{10-16}$ that in this case the main contributions to the Hall current will be those from impurities via the side jump and the skew scattering mechanisms. In contrast to the extrinsic AHE, the intrinsic one assumes that the spin-orbit coupling is already present in the band structure of the system and generally cannot be considered as weak in comparison even with the Fermi energy. Recently the interest toward the intrinsic AHE has grown up considerably due to different applications in the diluted magnetic semiconductors (DMS) and due to the interesting modern interpretation of the Hall current in terms of the Berry phase. ${ }^{5,17}$ The Berry phase contribution to the Hall current was shown to be in good quantitative agreement with experiment in many different materials with strong spin-orbit couping in their band structure, giving weight to the theory involving this contribution alone. ${ }^{17-19}$

The Luttinger's theory of the intrinsic AHE, however, predicts that other contributions should arise due to the scattering from a disordered potential even if the disorder potential itself is spin independent. The asymmetry in scatterings becomes transparent in the basis related to the Bloch states. As an example, Luttinger considered a rather simplified model which demonstrated that such corrections must reverse the sign of the Hall current in the dc limit, in comparison to the clean limit, though in the high-frequency ac case the clean limit contribution should dominate. The same results have been shown by several other works utilizing different approaches and focusing on this simplified model. ${ }^{20-22}$

The results from these simplified models seem to be in a contradiction with recent work which did not find such a change of sign in numerical simulations or in a comparison with the experimental results. We show here that a possible resolution of this discrepancy is the simplicity of the early models from which many generalizations were stated without justification and whose results depended drastically on the simple momentum dependence of the Berry curvature of the Bloch states.

The work by Luttinger and works of other authors, related to the disorder contribution to the AHE, are rather involved. Generally various contributions separately turned out to be not gauge invariant and only the final result was physically meaningful. Because of these shortcomings, we reformulate the basic arguments of the previous authors in terms of wave-packet dyanamic equations which are fully gauge invariant and consistent with prior results. ${ }^{6}$ Being gauge invariant, the wave-packet equations allow one to identify the physical meaning of various contributions. We should note, however, that the wave-packet equations are valid only in the limit of smooth potentials. This restricts the applicability of 
our conclusions and generalizations of our results should not be made to regimes where the semiclassical treatment is not justified without careful checks; therefore we did not make the goal to construct the final theory of the disorder in the AHE but rather to construct a simple formalism that demonstrates basic features of the problem and highlights a basic key ingredient missed by prior theories studying the effects of disorder, namely the importance of the momentum dependence of the Berry curvature.

We have found that the change of the sign of the Hall current due to the disorder, found in the early works, is not universal and arises in the models where the bands have constant Berry curvature in momentum space. We focus our attention to the case of Rashba coupled two-dimensional electron gas (R2DEG) with smooth disorder, where the timereversal symmetry is broken by an out-of-plane Zeeman field. In the diluted magnetic semiconductors the Berry curvature is strongly momentum dependent. This separates parametrically the clean contribution from the others and makes the sign reversal not universal. Rather generally we find the same sign of the total Hall current for realistic bands except in extreme situations where one of the bands becomes depleted.

We organize the rest of the paper as follows. In Sec. II we review the wave-packet dynamics theory of Sundaram and Niu. ${ }^{6}$ In Sec. III we analyze the model for constant Berry curvature obtaining in a physically clear way the reversal of sign in the presence of smooth disorder. In Sec. IV we apply the theory to the case of the Rashba model and show the nontrivial dependence of the contributions from disorder scattering and the clean Berry phase contribution as a function of the Fermi energy, and in Sec. V we present our conclusions.

\section{WAVE-PACKET EQUATIONS}

The motion of wave packets formed by Bloch states is governed by the following equations: ${ }^{6}$

$$
\begin{gathered}
\frac{d}{d t} \mathbf{k}=e \mathbf{E}-\nabla V(\mathbf{r}), \\
\frac{d}{d t} \mathbf{r}=\frac{\partial \epsilon(\mathbf{k})}{\partial \mathbf{k}}-\frac{d \mathbf{k}}{d t} \times \mathbf{F},
\end{gathered}
$$

where $\boldsymbol{\epsilon}(\mathbf{k})$ is the energy dispersion in the band, $\mathbf{E}$ is the external electric field acting on a wave packet having the electric charge $e, V(\mathbf{r})$ is the local potential in the sample, for example, the potential of impurities, and $\mathbf{F}$ is the Berry curvature of the Bloch band. For the two-dimensional motion only the out-of-plane component of $\mathbf{F}$ is nonzero,

$$
F_{z}=2 \operatorname{Im}\left\langle\frac{\partial u^{s}}{\partial k_{y}} \mid \frac{\partial u^{s}}{\partial k_{x}}\right\rangle,
$$

where $\left|u^{s}\right\rangle$ is the Bloch state in the absence of the electric field and impurities and $s$ is the index of the band.

The second term in Eq. (2) is responsible for the so-called anomalous velocity. For the reader not familiar with the notion of anomalous velocity we provide in the Appendix a simple example from classical physics that gives an intuitive explanation of the physical meaning of the anomalous velocity in Rashba coupled 2DEG. For the rigorous theory we refer to the original papers. ${ }^{6}$

The anomalous velocity is orthogonal to the direction of the electric field ${ }^{1,23}$ (which we chose to be along the $x$ axis),

$$
v_{y}^{(a)}=F_{z} \frac{d k_{x}}{d t} .
$$

The semiclassical equations (1) and (2) map the quantummechanical problem to a classical one where particles have the electric charge $e$ and move according to equations of the wave-packet dynamics. We assume that at equilibrium the distribution of such classical particles is the same as the Dirac distribution of electrons in the sample. This mapping to a classical system considerably simplifies the treatment of the problem both analytically and numerically. Analytically, one can apply the classical Boltzmann equation approach to calculate the transport coefficients, numerically the molecular-dynamics simulation of the motion of classical particles is simple and may not be restricted to a small system size as, for example, in the case of a numerical diagonalization of a quantum-mechanical Hamiltonian.

Unfortunately Eqs. (1) and (2) are valid in the adiabatic limit only. They can be applied to very smooth impurity potentials so that transitions between bands can be disregarded. They cannot be applied to the case of scatterings on a short-range delta-function-like potential. Only scatterings on impurities, whose potential varies appreciably only on distances much larger than the size of the wave packet, can be calculated this way. ${ }^{6}$ In spite of this restriction of its applicability the limit of a smooth potential is a very interesting one to investigate the influence of disorder on the anomalous Hall effect. In realistic applications a system with long-range impurities is realized, for example, in the high mobility 2D electron gas $^{24}$ and the out-of-plane Zeeman field can be induced there by polarizing nuclear spins or by introducing additional magnetic impurities.

Recently another related effect, namely the intrinsic spin Hall effect, was introduced. ${ }^{25}$ A number of theoretical papers have explored the importance of the disorder. The debates on this topic are ongoing (see, e.g., Refs. 26-28). Understanding the AHE may shed light on the disorder role in the spin Hall effect.

\section{CONSTANT BERRY CURVATURE}

\section{A. Clean limit}

In many recent applications the Berry curvature $\mathbf{F}$ strongly depends on the momentum of the wave packet $k^{5,19,30}$ However, it is instructive to consider first the one Bloch band 2D system with constant Berry curvature $F_{z}$. The case with constant Berry curvature has been considered in the earlier theories of the AHE, whose results have been extrapolated directly to systems with nontrivial Berry curvature momentum dependence. We show in the following section that such extrapolation is unjustified in the cases where the Berry curvature has a strong dependence on momentum. 
This, as we will show, leads to interesting predictions for R2DEG and probably DMS.

For a constant $F_{z}$ the anomalous velocity (4) leads to the following contribution to the Hall current from the unperturbed part of the distribution function in a single band at zero temperature:

$$
\begin{aligned}
J_{y x}^{(\text {clean })} & =e \int d^{2} \mathbf{k} f_{0}(\mathbf{k}) v_{y}^{(a)}=e \int_{0}^{k_{F}} k d k \int_{0}^{2 \pi} \frac{d \phi}{(2 \pi)^{2}} e E_{x} F_{z} \\
& =\frac{e^{2} E_{x} F_{z} k_{F}^{2}}{4 \pi},
\end{aligned}
$$

where $f_{0}(\mathbf{k})$ is the equilibrium distribution function and $k_{F}$ is the Fermi momentum. The upper index (clean) means that the quantity is calculated in the absence of disorder and the distribution function of the wave packets coincides with the one in equilibrium at $E_{x}=0$.

The case of constant $F_{z}$ is realized in the conducting bands whose states are weakly hybridized with the states in the valent hole bands ${ }^{21}$ where the spin-orbit coupling is allowed. If the kinetic energies of electrons in the conducting band are much smaller than the gap between conducting and valent zones, the Berry curvature due to such an induced spin-orbit coupling can be considered as a constant for all conducting electrons. In modern applications it can be realized in some limiting situations, like in the case of R2DEG at a strong Zeeman field (see the following sections). The main conclusion in the early literature was that due to the spinorbit coupling, in the stationary state the distribution function acquires an additional asymmetric contribution plus the socalled side jump, namely the shift of the electrons during the impurity scattering changes the average drift velocities so that the total Hall current reverses the sign in comparison with Eq. (5) and has the same absolute value. In this section we show that for smooth impurity potentials the wave-packet approach easily reproduces the sign reversal of the Hall current when $F_{z}=$ const. In comparison to previous works, however, our approach keeps the derivation gauge invariant and hence is physically clear.

\section{B. Effects of disorder}

According to Eq. (5) if the distribution function is the same as in the equilibrium at zero external field a Hall current appears in the external field due to the anomalous velocity. However, in the steady state the distribution function is no longer $f_{0}(\mathbf{k})$. In the electric field and on time scales much larger than the scattering time particles diffuse with a constant velocity rather than accelerate as in the absolutely clean case. The anomalous velocity and hence the clean contribution to the Hall current are proportional to the acceleration $\dot{k}_{x}$. Since in the steady state the average acceleration is zero up to the first order in external electric field $E_{x}$ one can expect that disorder should strongly influence the Hall current.

A natural way to study the effect of disorder on the transport in our case is the classical Boltzmann equation. There is, however, a complication when both nonzero Berry curvature and finite sizes of impurities must be considered. At a scat- tering on an impurity potential not only the momentum but also the coordinate of a particle changes. Usually such a coordinate shift at the scattering is discarded since after averaging over many scatterings such random shifts cancel each other and only the changes of the momentum matter. However, when Berry curvature is nonzero the additional (anomalous) shift does not disappear after the averaging. To see this, suppose that the term with the Berry curvature $F_{z}$ in Eq. (2) is small in comparison with the first one and calculate the corresponding correction to the shift of the particle during the scattering on impurity. Integrating Eq. (2) over the time interval at which a particle feels the impurity potential during a single scattering and treating the second term in Eq. (2) as a small perturbation one can find that, after the scattering, a particle makes an additional shift,

$$
\Delta \mathbf{r}\left(\mathbf{k}, \mathbf{k}^{\prime}\right)=\mathbf{z} \times\left(\mathbf{k}^{\prime}-\mathbf{k}\right) F_{z}+\int_{t_{1}}^{t_{2}} \frac{\partial \epsilon}{\partial \mathbf{k}} d t,
$$

where $\mathbf{k}^{\prime}$ and $\mathbf{k}$ are momentums, respectively, after and before the scattering, $t_{1}$ and $t_{2}$ are times of entering and leaving the impurity in a semiclassical picture. The second term on the right-hand side of Eq. (6) is just the shift due to the normal velocity. To first order in $F_{z}$ and $\nabla V$ it is not affected by the Berry phase and hence is averaged to zero after many scatterings; therefore we will disregard it in our future discussion.

The particle's displacement due to the first term in Eq. (6) is due to the anomalous velocity. This shift does not depend explicitly on the details of the impurity potential and does not have the chiral symmetry. There are two main rather distinct effects due to the appearance of this anomalous shift. The first effect, the so-called side jump, is that the $y$ component of this shift does not cancel after the averaging over many scatterings and thus contributes to the drift velocity perpendicular to the electric field, i.e., to the Hall current. We will focus on this effect in the following subsection. The second effect is that when a scattering takes place in the presence of an external electric field there is a change in the potential energy upon a scattering given by

$$
\Delta U=-e E_{x} \Delta x,
$$

where $\Delta x$ is the shift along the external electric field. ${ }^{11-13}$ Both of these effects, as shown below and in the next subsection, give the same contribution as Eq. (5) but with an opposite sign in the particular case of a momentumindependent Berry curvature. Because both contributions are linear in $E_{x}$ we are able to consider them separetly when considering the linear response of the system.

Let us focus first on the second effect. Here only the anomalous part of this shift is important for Hall current calculations,

$$
\Delta x=-F_{z}\left(k^{\prime}{ }_{y}-k_{y}\right),
$$

and the effect of the normal part of the shift only renormalizes the diagonal current response to $E_{x}$. Since the total energy remains the same after an elastic scattering, the kinetic energy must change by the same amount,

$$
\Delta \epsilon\left(\mathbf{k}, \mathbf{k}^{\prime}\right)=\epsilon\left(\mathbf{k}^{\prime}\right)-\epsilon(\mathbf{k})=e E_{x} \Delta x .
$$


This effect leads to an instability of the initial equilibrium distribution function $f_{0}(\mathbf{k})=f_{0}(\epsilon(\mathbf{k}))$ due to scatterings. The Boltzmann equation is given by

$$
\frac{\partial f}{\partial t}=-\sum_{\mathbf{k}^{\prime}} \omega\left(\mathbf{k}, \mathbf{k}^{\prime}\right)\left(f(\epsilon(\mathbf{k}))-f\left(\epsilon\left(\mathbf{k}^{\prime}\right)\right)\right),
$$

where $\omega\left(\mathbf{k}, \mathbf{k}^{\prime}\right)$ is the scattering rate. Kinetic energies before and after a scattering do not coincide but the difference between them is small. Since we are seeking a contribution linear in $E_{x}$ we can substitute $f$ by $f_{0}$ which depends only on the kinetic energy $\epsilon(\mathbf{k})$ so we can expand

$$
\begin{aligned}
f_{0}(\epsilon(\mathbf{k}))-f_{0}\left(\epsilon\left(\mathbf{k}^{\prime}\right)\right) & =\frac{-d f_{0}}{d \epsilon}\left[\epsilon\left(\mathbf{k}^{\prime}\right)-\epsilon(\mathbf{k})\right] \\
& =-\frac{d f_{0}}{d \epsilon} e E_{x} F_{z}\left(k_{y}-k^{\prime}{ }_{y}\right) .
\end{aligned}
$$

This yields

$$
\frac{\partial f}{\partial t}=-\sum_{\mathbf{k}^{\prime}} \omega\left(\mathbf{k}, \mathbf{k}^{\prime}\right) \frac{-d f_{0}}{d \epsilon} e E_{x} F_{z}\left(k_{y}-k_{y}^{\prime}\right) \neq 0 .
$$

The distribution function will relax until a contribution to it compensates this relaxation in the stationary limit.

To find the new equilibrium distribution one should substitute $f(\mathbf{k})=f_{0}(\epsilon(\mathbf{k}))+g_{E}(\mathbf{k})$ into the right-hand side of Eq. (10). To make $\partial f / \partial t=0$ in Eq. (12) the correction $g_{E}(\mathbf{k})$ must be the following:

$$
g_{E}(\mathbf{k})=-\frac{-d f_{0}}{d \epsilon} e E_{x} k_{y} F_{z} .
$$

This contribution is not symmetric in the $y$ direction. This means that already the normal velocity can contribute to the Hall current in the stationary state:

$$
J_{y x}^{\text {normal }}=e \int \frac{d^{2} \mathbf{k}}{(2 \pi)^{2}} g_{E}(\mathbf{k})\left(v_{y}^{(\text {normal })}\right)=-\frac{e^{2} E_{x} F_{z}}{(4 \pi)} k_{F}^{2}
$$

which has the opposite sign and is exactly the same in the absolute magnitude as the anomalous velocity contribution in the clean limit given by Eq. (5).

\section{Side jump}

The anomalous change of energy after the scattering is not the only important effect of the anomalous shift (7). The anomalous shift during the scatterings has generally a component perpendicular to the direction of the electric field,

$$
\Delta y\left(\mathbf{k}, \mathbf{k}^{\prime}\right)=F_{z}\left(k_{x}^{\prime}-k_{x}\right) .
$$

It does not contribute to the change of energy during the scattering but it shifts a particle along the $y$ axes. If such shifts do not compensate each other after many scatterings, they should contribute to the total Hall current. This phenomenon is known in the theory of the extrinsic Hall effect as the side jump. If scatterings happen with the rate $\omega\left(\mathbf{k}, \mathbf{k}^{\prime}\right)$ in average the particle moving with the momentum $\mathbf{k}$ also acquires the anomalous drift velocity perpendicular to the electric field,

$$
\left\langle v_{y}^{(s j)}(\mathbf{k})\right\rangle_{\mathrm{imp}}=\sum_{\mathbf{k}^{\prime}} \omega\left(\mathbf{k}, \mathbf{k}^{\prime}\right) \Delta y\left(\mathbf{k}, \mathbf{k}^{\prime}\right)=-F_{z} k_{x} / \tau_{\|},
$$

where

$$
1 / \tau_{\|}=\sum_{\mathbf{k}^{\prime}} \omega\left(\mathbf{k}, \mathbf{k}^{\prime}\right)\left[1-\cos \left(\mathbf{k}, \mathbf{k}^{\prime}\right)\right]
$$

and " $(s j)$ " marks the side jump contribution to the physical quantity.

In the equilibrium the side jump does not lead to the Hall current because the anomalous velocity (16) changes the sign under the transformation $k_{x} \rightarrow-k_{x}$. and the distribution function in the equilibrium is invariant under this transformation. However, when the electric field is applied the nonequilibrium correction to the distribution function appears which has no such symmetry under the momentum reflection.

This correction to the distribution function can be derived by means of the standard approach to the Boltzmann equation. Up to the first order in the electric field the standard Boltzmann equation for one band, ignoring the asymmetric contribution considered in the previous subsection, is

$$
-e E_{x} v_{x}^{(\text {normal })}\left(-\frac{\partial f_{0}}{\partial \epsilon}\right)=-\sum_{\mathbf{k}^{\prime}} \omega\left(\mathbf{k}, \mathbf{k}^{\prime}\right)\left[f(\mathbf{k})-f\left(\mathbf{k}^{\prime}\right)\right]
$$

where $f_{0}(\mathbf{k})$ is the equilibrium distribution function. Equation (18) has a solution $f(\mathbf{k})=f_{0}(\mathbf{k})+g(\mathbf{k})$ where to the first order in the electric field

$$
g(\mathbf{k})=e E_{x} \tau_{\|}\left(-\frac{\partial f_{0}}{\partial \epsilon}\right) v_{x}^{(\text {normal })},
$$

$v_{x}^{\text {(normal) }}$ is the normal velocity along the electric field, which in our case is

$$
v_{x}^{(\text {normal })}=\frac{\partial \epsilon(k)}{\partial k_{x}}=b k_{x},
$$

where we assumed that the conducting band has a trivial dispersion $\epsilon(\mathbf{k})=b k^{2} / 2$ where $b=1 / m_{e}$. The side-jump contribution of impurities to the anomalous velocity leads to the Hall current

$$
\begin{aligned}
J_{y x}^{(s j)} & =e \int\left\langle v_{y}^{(s j)}(\mathbf{k})\right\rangle_{\mathrm{imp}} g(\mathbf{k}) \frac{k d k d \phi}{(2 \pi)^{2}} \\
& =-\frac{e^{2} E_{x} F_{z}}{(2 \pi)^{2}} \int_{0}^{2 \pi} d \phi \cos ^{2}(\phi) \int d \epsilon_{k} k^{2} \delta\left(\epsilon_{F}-\epsilon_{k}\right) \\
& =-\frac{e^{2} E_{x} F_{z}}{(4 \pi)} k_{F}^{2} .
\end{aligned}
$$

As in the previous impurity contribution, the Hall current due to the side-jump has the opposite sign and the same magnitude as the one in the clean limit. The physical meanings of both impurity contributions, however, are very different. The side jump current appears because the anomalous velocity is proportional to the acceleration of the particle but in the stationary state the average acceleration should be zero (up to the terms of the first order in the electric field), 
namely, while the external electric field accelerates the wave packet during its motion between impurities, the impurity potential generally decelerates it. During such a deceleration the wave packet has the anomalous velocity with the opposite sign and hence the side-jump contribution should compensate or at least decrease the pure limit result. The described picture is of course oversimplified, which will be clear from the discussion of a momentum dependent $F_{z}$. In reality, the external electric field equally accelerates all electrons in the Fermi sea but effective deceleration due to impurity scatterings is, on average, seen only by the electrons near the Fermi level because there the distribution function acquires a correction required to compensate the acceleration by the external field. Hence the side-jump contribution is the property of the Fermi surface while the clean limit Berry phase contribution appears from all electrons even deep in the Fermi sea.

\section{Total current and numerical check}

The total Hall current is the sum of the clean limit contribution, of the side-jump and of the normal velocity contributions. For the constant $F_{z}$ we find

$$
J_{y x}^{(\text {total })}=J_{y x}^{(\text {clean })}+J_{y x}^{(s j)}+J_{y x}^{(\text {normal })}=-\frac{e^{2} E_{x} F_{z}}{(4 \pi)} k_{F}^{2} .
$$

As it was predicted in the former literature which focused on models with constant $F_{z}$, the total current has the same magnitude but with the opposite sign as the one in the absolutely clean system. Our derivation, however, is considerably simpler and is straightforward to generalize to a case with a more complicated band structure such as Rashba coupled 2DEG.

To confirm the analytical result (22) numerically we simulate the motion of particles according to equations of motions (1) and (2). Initially all particles were prepared uniformly distributed over the momentum space having the absolute value of the momentum less than a specified Fermi momentum. The action of an impurity was simulated by the potential of a wall of a finite thickness with a very fast linearly growing potential inside the wall. The wall is sufficiently long to disregard the effects on its edges. This type of impurity does not allow the skew scattering mechanism to appear because for every scattering on the wall that changes momentum from $\mathbf{k}$ to $\mathbf{k}^{\prime}$ there is a process when a particle hits the same wall from another side and scatters from $\mathbf{k}^{\prime}$ into $\mathbf{k}$. Thus both processes have equal probability and $\omega\left(\mathbf{k}, \mathbf{k}^{\prime}\right)$ $=\omega\left(\mathbf{k}^{\prime}, \mathbf{k}\right)$. Figure 1 demonstrates a scattering on such an impurity. We would like to point to the analogy of the scattering in Fig. 1 with Goos-Hänchen's and with Fedorov's shifts in geometrical optics. ${ }^{29}$ One can consider the effect of a wall on the wave packet as the one due to an electric field $E_{\perp}$ acting on it only inside the wall in the direction perpendicular to the wall. Although the potential in the wall can be strong in comparison with the external electric field, we suppose that the motion inside the wall is still governed by the equations of the wave-packet dynamics. Such a scattering problem can be solved exactly. This solution shows that for the given type of scatterings the theory of the anomalous

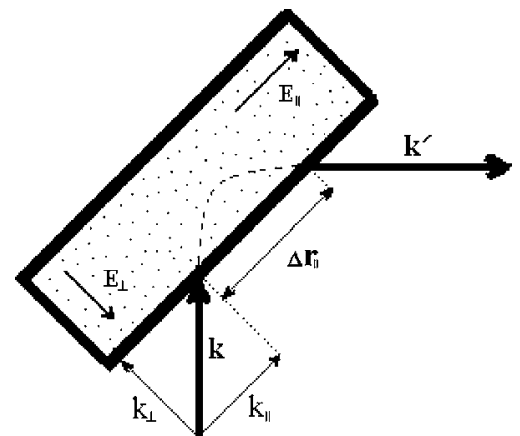

FIG. 1. Scattering of a particle on impurity. The impurity is assumed to have the shape of a long stripe with linearly growing potential inside.

shift is valid even if we do not assume that the anomalous velocity term in wave-packet equations is small. This is useful because the bigger anomalous velocity is needed to accelerate the numerical calculations.

Let components of the momentum of the particle incident on the wall be $k_{\|}$and $k_{\perp}$ parallel and perpendicular to the wall, respectively. In the presence of an additional external electric field the total force has generally also a nonzero component parallel to the wall $e E_{\|}$where $E_{\|}$is the projection of the external electric field on the direction along the wall. Expressions for the final momentum and coordinates right after the scattering are strongly simplified in the limit of large $E_{\perp}$, so that all terms $O\left(1 / E_{\perp}\right)$ can be dropped. In this limit the scattering time is vanishing and in Eq. (2) one can disregard the first term in comparison with the second one since $\dot{k}_{\perp} \sim E_{\perp}$ is large. Dropping the term with $\partial \epsilon / \partial k_{\perp}$ Eq. (2) can be readily integrated over the time of the scattering leading to the relations $\Delta r_{\|}=e F_{z}\left(k_{\perp}-k^{\prime}{ }_{\perp}\right)$ and $k_{\|}^{\prime}=k_{\|}$. Solving them together with the energy conservation equation $\epsilon(\mathbf{k})+e E_{\|} r_{\|}=\epsilon\left(\mathbf{k}^{\prime}\right)$ we arrive at following expressions for the momentum of the outgoing particle:

$$
\begin{gathered}
k_{\|}^{\prime}=k_{\|} \\
k_{\perp}^{\prime}=-k_{\perp}-E_{\|} F_{z} .
\end{gathered}
$$

In addition, the scattered particle appears in a point of the interface shifted in comparison to the incident point by the amount

$$
\Delta r_{\|}=2 k_{\perp} F_{z}+E_{\|} F_{z}^{2} .
$$

The shift $r_{\|}$is exactly the anomalous shift in the impurity potential. Note also that, as it follows from Eq. (23), when a scattering happens in the additional external electric field with nonzero projection to the direction of this shift $E_{\|}$, the absolute magnitude of the momentum changes, which leads to the instability of the equilibrium distribution function, discussed in previous sections. The scattering time in this limit is small enough to be disregarded so numerically such a scattering can be easily simulated by choosing the coordinate system related to the wall orientation and updating the momentums and coordinates according to $\left(k_{\|}, k_{\perp}, r_{\|}, r_{\perp}\right)$ $\rightarrow\left(k_{\|}^{\prime}, k_{\perp}^{\prime}, r_{\|}+\Delta r_{\|}, r_{\perp}\right), r_{\perp}$ is the same for the incident and 


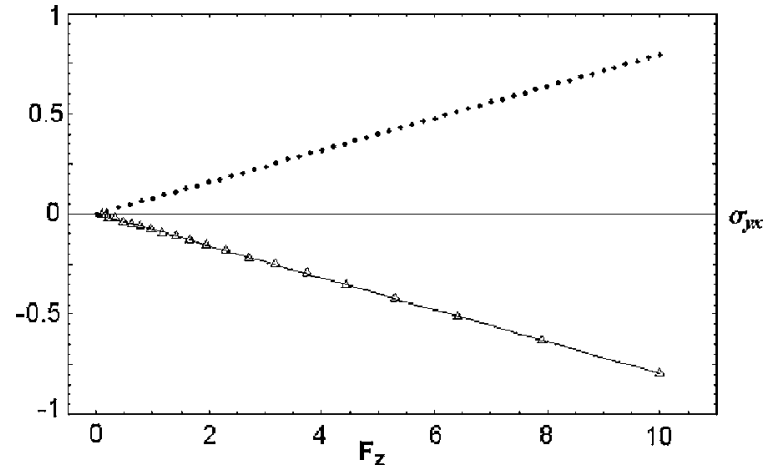

FIG. 2. Hall conductivity vs strength of the Berry curvature in the model with momentum independent $F_{z}$. Solid line is the theoretical prediction that includes impurity scatterings and dotted line is the prediction of the clean limit contribution. Triangles show results of the numerical simulations. Simulations were performed in units $e=\hbar=m=1$ and $k_{F}=1$.

the outgoing particle. In between scatterings equations of free motion in the external electric field are also trivially solvable. In our simulations we assumed the "noncrossing approximation," namely we suppose that every scattering happens on a different impurity. The algorithm consists of two parts. First we generate randomly the distance $L$ to the next impurity. We suppose that between impurities particles move only under the action of the electric field. The integration of wave-packet equations leads to the following trajectories:

$$
\begin{gathered}
k_{x}^{\prime}=k_{x}+E_{x} t, \\
k^{\prime}{ }_{y}=k_{y}, \\
x^{\prime}=x+k_{x} t+\frac{E_{x} t^{2}}{2}, \\
y^{\prime}=y+\left(k_{y}+F_{z} E_{x}\right) t .
\end{gathered}
$$

The time $t$ of the motion to the next impurity was estimated from the equation $\left[\left(x^{\prime}-x\right)^{2}+\left(y^{\prime}-y\right)^{2}\right]^{1 / 2}=L$. Solving this relation with the solution (25) we find the momentum and coordinates of the particle before the new scattering. In the second step we randomly generate the orientation angle of the impurity, switch into the related coordinate system, and update particle's coordinates and the momentum according to Eqs. (23) and (24). Then we return to the initial coordinate system and repeat the circle. We repeat this circle sufficiently many times to allow the distribution to relax to the stationary one (usually a few scatterings is enough), however, the total evolution time was small enough in order to avoid strong heating of the system. In our simulations, every particle makes about $10^{2}-10^{3}$ scatterings during the whole time. To prevent strong heating the electric field is chosen sufficiently small $E_{x} l / E_{F} \sim 10^{-4}$ where $l$ is the typical scattering length and $E_{F}$ is the Fermi energy. The total current can be derived as the sum of total displacements of all particles divided by the evolution time.

In Fig. 2 we compare the analytical result (22) with our

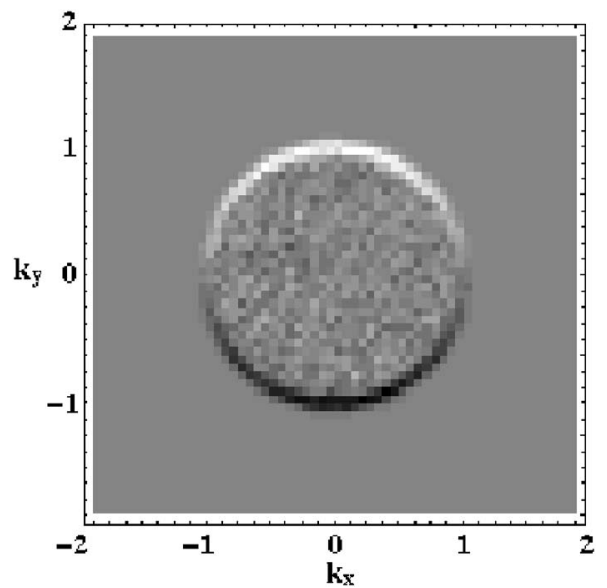

FIG. 3. Distribution of particles over the momentum space in the stationary state with electric field applied along the $y$ axis at zero Berry curvature. Parameters are as follows: $k_{F}=1, E_{y}=0.003$, $E_{x}=0, F_{z}=0$. Average distance between impurities is $l \sim 0.5$ in units $m=\hbar=e=1$.

numerical simulations for the fixed Fermi energy but different strengths of the spin-orbit coupling, and hence $F_{z}$. Both analytical and numerical results are in excellent agreement with each other. This result survives at an arbitrary magnitude of the Berry curvature.

In Figs. 3 and 4 we visualized the stationary distribution function of particles in the momentum space, calculated numerically for the system placed in the uniform electric field. Brighter areas represent higher densities. To increase the contrast, we subtracted the initial equilibrium distribution (i.e., without electric field) from the calculated one. In Fig. 3 the Berry curvature is set to zero. In this case the correction to the equilibrium distribution is symmetric along the electric field. Figure 4 shows that when the Berry curvature becomes nonzero, the distribution function acquires the additional asymmetric contribution, which obviously deposits to the Hall current.

\section{E. Analogy between extrinsic and intrinsic AHE}

As we showed above, in the AHE there is a possibility of disorder effects similar to those previously studied in the extrinsic AHE studies focused on gradient terms of the disorder but arising instead from Berry curvature considerations. In this subsection we discuss how this relation is encoded in the wave-packet equations (1) and (2). We consider here only the case of constant Berry curvature. The wavepacket dynamic equations do not arise from a particular Hamiltonian. Nevertheless, it is possible to make them arise from a Hamiltonian by adding terms of higher order in potential gradient. We remind the reader that Eqs. (1) and (2) were derived in the approximation of a smooth potential so that its higher gradients could be discarded. Therefore such a procedure should not change the physics at least to the order of the approximation of the whole theory. Consider the following system:

$$
\frac{d}{d t} \mathbf{k}=e \mathbf{E}-\nabla V(\mathbf{r})-\nabla[\mathbf{k} \times \nabla V(\mathbf{r})] \mathbf{F}
$$




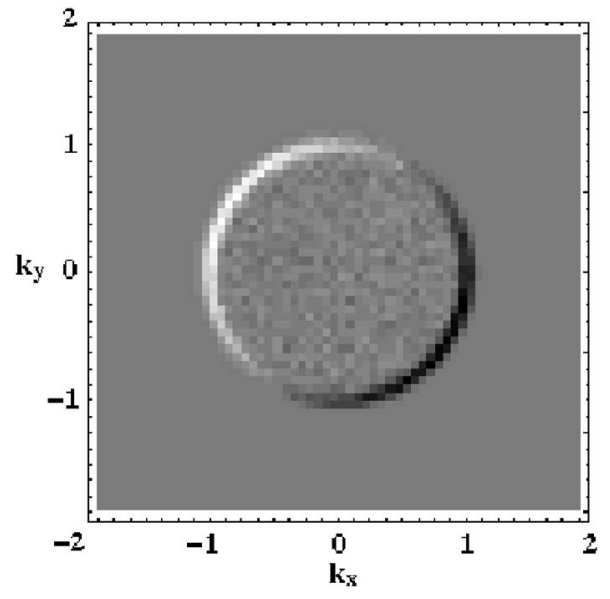

FIG. 4. Distribution of particles over the momentum space in the stationary state with electric field applied along the $y$ axis at the nonzero Berry curvature. Parameters are as follows: $k_{F}=1, E_{y}$ $=0.003, E_{x}=0, F_{z}=-1$. Average distance between impurities is $l$ $\sim 0.5$.

$$
\frac{d}{d t} \mathbf{r}=\frac{\partial \epsilon(\mathbf{k})}{\partial \mathbf{k}}-[e \mathbf{E}-\nabla V(\mathbf{r})] \times \mathbf{F} .
$$

Except for one term of the second order in gradients of the potential the system is equivalent to the wave-packet equations (1) and (2). The new system (26) and (27) already corresponds to the classical Hamiltonian evolution with the following Hamiltonian:

$$
H(\mathbf{k}, \mathbf{r})=\boldsymbol{\epsilon}(\mathbf{k})-\mathbf{F}(\mathbf{k} \times[e \mathbf{E}-\nabla V(\mathbf{r})])+V(\mathbf{r})-e \mathbf{E r} .
$$

This Hamiltonian has the same structure as in typical models of the extrinsic AHE, ${ }^{11-13}$ and the Berry curvature now plays the role of an effective spin-orbit coupling constant. As we mentioned, the main contributions to the extrinsic Hall current have been proved to be the side jump and the skew scattering. In our previous calculations, including numerics, we ignored the skew scattering mechanism, as it is rather different parametrically, nevertheless the skew scattering can be calculated by same wave-packet techniques because the second term on the right-hand side of Eq. (28) is responsible for both skew scattering and the side-jump contributions in the extrinsic AHE. Note also that the skew scattering current will depend on the Berry curvature and thus will also have a geometric interpretation. The fact that the side jump is the only other surviving contribution in the extrinsic AHE is in agreement with our previous findings.

\section{RASHBA 2DEG WITH EXCHANGE COUPLING}

Recently studied systems with AHE such as DMS and Rashba 2DEG have Berry curvature that strongly depends on momentum of the wave packet. ${ }^{30}$ When $F_{z}$ is no longer a constant the simple arguments leading to canceling of some terms may not work. One can notice that when an electron decelerates it has in general different momentum than when it accelerates, in other words, the uniform electric field ac- celerates all electrons down to the bottom of the Fermi sea but impurities produce nonzero deceleration in average only for electrons near the Fermi surface. The Berry phase acquired by accelerating electrons depends now not only on the acceleration but on the momentum itself, therefore contributions from impurities and from uniform electric field do not necessarily cancel each other.

The Hamiltonian of R2DEG with the electric field along the $x$ direction and the exchange field in the $z$ direction is

$$
\begin{gathered}
H=H_{0}+H_{S O}+H_{e x c h}-e E_{x} x+V_{i m p}, \\
H_{0}=\frac{b k^{2}}{2} \sigma_{0}, \quad b=1 / m, \\
H_{S O}=\lambda\left(k_{y} \sigma_{x}-k_{x} \sigma_{y}\right), \\
H_{\text {exch }}=h \sigma_{z},
\end{gathered}
$$

where $V_{\text {imp }}$ is the impurity potential.

Diagonalizing the unperturbed part of the Hamiltonian we find the energy dispersion

$$
\epsilon_{ \pm}(k)=b k^{2} \pm \sqrt{(\lambda k)^{2}+h^{2}} .
$$

There are two bands; the minor band has the plus sign in the above expression. Fermi momentums can be derived by inversion of Eqs. (33). We denote them as $k_{F-}$ and $k_{F+}$, respectively, for the major and minor bands.

The Berry curvature is different for different bands,

$$
F_{z}^{s}=2 \operatorname{Im}\left\langle\frac{\partial u^{s}}{\partial k_{y}} \mid \frac{\partial u^{s}}{\partial k_{x}}\right\rangle,
$$

where $\left|u^{s}\right\rangle$ is the Bloch state in the absence of electric field and impurities and $s= \pm$ is the index of the band: the plus is for the minor and the minus is for the major one. In the case of the Hamiltonian (29) the Berry curvature is ${ }^{30}$

$$
F_{z}^{s}(k)=-s \frac{h \lambda^{2}}{2\left[(\lambda k)^{2}+h^{2}\right]^{3 / 2}},
$$

at $\lambda k \ll h$ this gives $F_{z}^{s}(k) \approx-s \lambda^{2} /\left(2 h^{2}\right)=$ const and we reduce the problem to the one considered in previous sections. In the opposite limit at $\lambda k \gg h$ we find

$$
F_{z}^{s}(k) \approx-\frac{s h}{2 \lambda k^{3}} .
$$

The anomalous velocity has the same magnitude but different signs for different bands so filled states with the same $\mathbf{k}$ from different bands will compensate each other in calculations of the clean contribution and to find the Hall current we should only integrate over uncompensated states of the major band. The Hall current from the unperturbed electron distribution function in the clean case is 


$$
\begin{aligned}
j_{y x}^{\text {(clean) }} & =e \int_{0}^{2 \pi} d \phi \int_{k_{F+}}^{k_{F-}} \frac{k d k}{(2 \pi)^{2}}\left[e E_{x} F_{z}^{-}(k)\right] \\
& =e \int_{0}^{2 \pi} d \phi \int_{k_{F+}}^{k_{F-}} \frac{k d k}{(2 \pi)^{2}} \frac{e E_{x} h \lambda^{2}}{2\left[(\lambda k)^{2}+h^{2}\right]^{3 / 2}} \\
& =\frac{e^{2} E_{x}}{2(2 \pi)}\left(\frac{1}{\sqrt{1+\left(k_{F+} \lambda / h\right)^{2}}}-\frac{1}{\sqrt{1+\left(k_{F-} \lambda / h\right)^{2}}}\right),
\end{aligned}
$$

in the case $E_{F} \gg \lambda k_{F \pm} \gg h$ the expression (37) is simplified,

$$
\begin{aligned}
j_{y x}^{\text {(clean) }} \approx & \left\{\lambda k \ll E_{F} \approx \frac{k_{F}^{2}}{2 m}\right\}=\frac{e^{2} E_{x} h\left(k_{F-}-k_{F+}\right)}{(2 \pi) 2 \lambda k^{2}} \\
& \times\left\{\left(k_{F-}-k_{F+}\right) \approx 2 \lambda m\right\}=\frac{e^{2} E_{x} h}{4 \pi E_{F}} ;
\end{aligned}
$$

note that here $k_{F+}$ is the Fermi momentum of the minor band. The above formulas are valid when there are electrons in both bands. At a sufficiently low Fermi level $\left(E_{F}<h, \lambda k_{F-}\right)$ the minor band becomes depleted and the transport is only in the major band. In this case the clean limit Hall current is

$$
j_{y x}^{\text {(clean) }}=\frac{e^{2} E_{x}}{2(2 \pi)}\left(1-\frac{1}{\sqrt{1+\left(k_{F-} \lambda / h\right)^{2}}}\right) .
$$

We address next the effects of impurity scattering within the formalism that we have developed.

As in the case of the constant Berry curvature, the nonequilibrium contribution to the distribution function is the sum of the usual one $g^{s}(\mathbf{k})$, which appears to compensate the electron acceleration

$$
g^{s}(\mathbf{k})=e E_{x} \tau_{\|}\left(-\frac{\partial f_{0}}{\partial \epsilon}\right) v_{s, x}^{(\text {normal })}
$$

and of the anomalous one $g_{E}^{s}(\mathbf{k})$ due to the shift of the kinetic energy of the scattered particle. We suppose here that the impurity potential is weak, namely $V(\mathbf{r}) \ll E_{F}, \lambda k_{F}$ and disregard the variation of the Berry curvature near a wave packet during a scattering because the absolute magnitude of the momentum does not change appreciably. The derivation of the anomalous correction is analogous to Eqs. (10)-(13),

$$
g_{E}^{s}(\mathbf{k})=-\frac{-d f_{0}}{d \epsilon} e E_{x} k_{y} F_{z}^{s} .
$$

The normal velocity of the wave packet is

$$
v_{s, x}^{(\text {normal })}=\frac{\partial \epsilon_{s}(k)}{\partial k_{x}} .
$$

The expression for the drift velocity due to the side jumps along the $y$ axis is the same as in Eq. (16). The side-jump current is

$$
\begin{aligned}
j_{y x}^{(s j)} & =\sum_{s= \pm} e \int\left\langle v_{s, y}^{(s j)}(\mathbf{k})\right\rangle_{d i s} g^{s}(\mathbf{k}) \frac{k d k d \phi}{(2 \pi)^{2}} \\
& =\sum_{s= \pm} \int d \phi \frac{\cos ^{2}(\varphi)}{(2 \pi)^{2}} \int d \epsilon_{s, k} e^{2} E_{x} F_{z}^{s}(k) k^{2} \delta\left(E_{F}-\epsilon_{s, k}\right) \\
& =\frac{h e^{2} E_{x} \lambda^{2}}{2(4 \pi)}\left(\frac{k_{F+}^{2}}{\left[\left(\lambda k_{F+}\right)^{2}+h^{2}\right]^{3 / 2}}-\frac{k_{F-}^{2}}{\left[\left(\lambda k_{F-}\right)^{2}+h^{2}\right]^{3 / 2}}\right) .
\end{aligned}
$$

At $E_{F} \gg \lambda k_{F} \gg h$ this expression can be simplified,

$$
j_{y x}^{(s j)} \approx \frac{h e^{2} E_{x}}{8 \pi E_{F}} .
$$

If $E_{F}<h$ only the major band survives and

$$
j_{y x}^{(s j)}=\frac{-h e^{2} E_{x} \lambda^{2}}{2(4 \pi)} \frac{k_{F-}^{2}}{\left[\left(\lambda k_{F-}\right)^{2}+h^{2}\right]^{3 / 2}} .
$$

The normal current contribution is

$$
\begin{aligned}
j_{y x}^{(\text {normal })} & =\sum_{s= \pm} e \int \frac{d^{2} \mathbf{k}}{(2 \pi)^{2}} v_{s, y}^{(\text {normal })} g_{E}^{s}(\mathbf{k}) \\
& =\sum_{s= \pm} \int d \phi \frac{\sin ^{2}(\varphi)}{(2 \pi)^{2}} \int d \epsilon_{s, k} e^{2} E_{x} F_{z}^{s}(k) k^{2} \delta\left(E_{F}-\epsilon_{s, k}\right) .
\end{aligned}
$$

Comparing the last expression with the one for the side-jump current (43) we find that they are different only by an exchange of $\cos (\varphi)$ and $\sin (\varphi)$ under the integral over the angle. Thus we arrive at the general result,

$$
j_{y x}^{(\text {normal })}=j_{y x}^{(s j)} .
$$

Finally,

$$
j_{y x}^{(\text {total })}=j_{y x}^{\text {(clean) }}+j_{y x}^{(s j)}+j_{y x}^{\text {(normal) }} .
$$

As it is seen from Eqs. (38) and (44) the side-jump contribution may not change the sign in comparison with the clean contribution for sufficiently large Fermi energy. This is very distinct from the case of the constant Berry curvature where such a contribution exactly cancels the pure one. At the limit $E_{F} \gg \lambda k_{F} \gg h$ we find for the total current

$$
j_{y x}^{(\text {total })} \approx \frac{h e^{2} E_{x}}{2 \pi E_{F}} .
$$

The total Hall current not only has the same sign as the clean limit prediction, but also increases due to scatterings. Note also that up to a coefficient of the order unity, in this limit, the total current has the same dependence on various parameters.

In Fig. 5 we compare the theoretical prediction of Eqs. (48) with the prediction of the pure Berry phase contribution (37).

\section{DISCUSSION AND CONCLUSION}

We calculated the anomalous Hall current in the presence of a smooth disorder potential by combining the wave-packet 


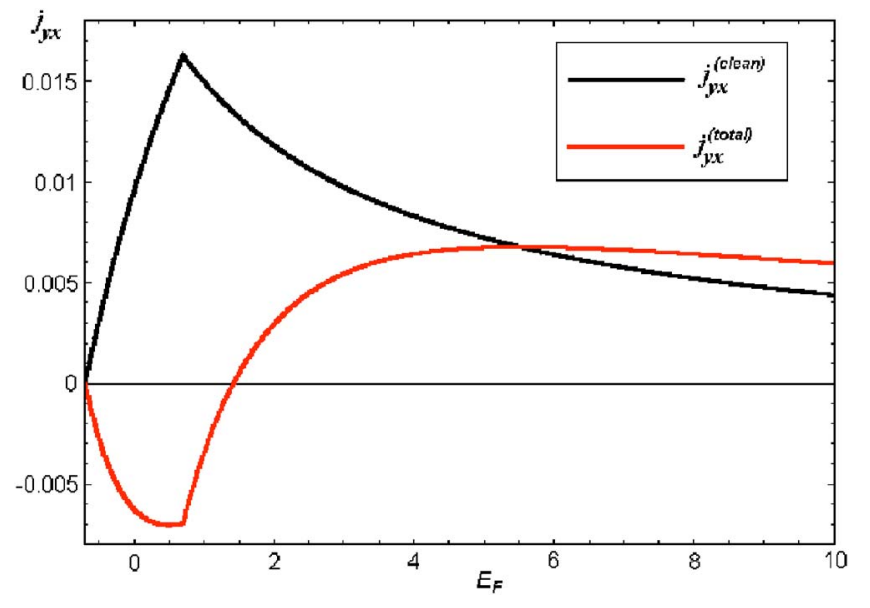

FIG. 5. (Color online) Hall conductivity vs the Fermi energy in the R2DEG. The black line is the pure limit Berry phase prediction. The red curve shows the prediction of the theory that includes impurity scatterings (43) and (47). Data are given in units $e=\hbar=m$ $=1$ for the case $h=0.7, \lambda=0.4$. The sharp change in the behavior above $E_{F}=0.7$ is due to the appearance of electrons in the minor band.

dynamic equations with the classical Boltzmann equation. For the case of constant Berry curvature our results are in agreement with some known predictions of the very earliest works on AHE. The gauge invariance of our approach clarifies the physical meaning of various disorder contributions to the Hall current and reveals the relation between the extrinsic, related to the gradients of the disorder potential, and Berry curvature effects in the presence of disorder.

We have shown that early results, like that of the Luttinger's sign reversal prediction, cannot be used directly for new applications such as diluted magnetic semiconductors, where the Berry curvature is strongly momentum dependent. Our prediction for the Hall current, Fig. 5, is distinct from the clean limit (or the pure Berry phase) one. Thus the total Hall current changes the sign when the Fermi level increases. In contrast, the clean limit prediction for the models considered remains of the same sign. The filled states in both bands with the same momentum compensate each other, hence for the clean case the band having more filled states always wins. In contrast, impurity contributions are sensitive not only to the density of states near the Fermi level, but also to the magnitude of the Berry curvature near the Fermi level.

The change of sign of the Hall current as a function of chemical potential originates from the competition between two bands. The minor band has the smaller number of filled states than the major one but it also has lower $k_{F}$ and hence the stronger $F_{z}\left(k_{F}\right)$. According to our calculations, such a change of sign should generally happen when the chemical potential is close to the depletion point of the minor band. When the minor band is totally depleted we always observe the reversal of the sign of the Hall current in comparison to the clean limit contribution. We also note that such a competition should be applicable to the skew scattering mechanism as well. This follows from the fact that the strength of the skew scattering should be proportional to the Berry curvature at the Fermi surface.
Our results can be generalized to DMS and although in this case the complicated behavior of the bands, as compared to the R2DEG, may change the quantitative behavior there is still a competition in DMS of Hall currents from major and minor bands and Berry curvature is also usually decreasing when momentum is increasing. However, the intricate dependence of the clean limit AHE observed in these systems versus the simple dependence observed in the R2DEG will require a careful analysis.

Although we have verified most of our predictions by numerical simulations, the correspondence between the wave-packet approach and the evolution of a true quantum system with disorder remains to be investigated. An effect not taken into account in our calculations is that the spin density in the wave packet polarizes when the wave packet is accelerated. ${ }^{31}$ This effect is crucial for the spin Hall effect and its importance for calculations by the Boltzmann equation should be understood.

The final theory of disorder in the AHE can be achieved only by purely quantum-mechanical calculations, for example, based on the Keldysh technique. At present interasting approaches to systems with Berry curvature have emerged, ${ }^{32}$ as well as different mechanisms to generate the anomalous Hall current, ${ }^{33}$ but we believe that the wavepacket approach combined with the classical Boltzmann equation is worth studying because it provides the simplest, to our knowledge, demonstration of the related physics and the wave-packet equations themselves have been well justified by the quantum theory.

Note added. Recently we became aware of the related effort by V. K. Dugaev et al. ${ }^{35}$ of a more quantummechanical approach to the problem with similar qualitative conclusions. Our approach, however, is quite different and in combination with this work may shed further light on the physical interpretation of the effect.

\section{ACKNOWLEDGMENTS}

The authors are grateful for insightful and useful discussions with A. H. MacDonald and V. L. Pokrovsky. This work was supported by the Welch Foundation, by DOE Grant No. DE-FG03-02ER45958 and by NSF under Grant No. DMR0115947.

\section{APPENDIX: CLASSICAL INTERPRETATION OF THE WAVE-PACKET DYNAMICS IN A RASHBA 2DEG}

To make the semiclassical result more transparent we show how the anomalous velocity arising from the Berry curvature can appear in a purely classical system. This classical anomalous velocity originates from the nonadiabatic contributions to the equations of motion in the linearresponse regime and are not present when a simpler adiabatic approximation is considered.

We consider the motion of a classical particle having the electric charge $e$ and the classical spin $\mathbf{S}$ attached to it. Choose the Hamiltonian to be 


$$
H=\frac{k^{2}}{2 m}+2 \lambda\left(k_{y} S_{x}-k_{x} S_{y}\right)-e E_{x} x+2 h S_{z} .
$$

The analogy with the Rashba Hamiltonian (29) is obvious. In the classical problem we substitute operators by corresponding classical variables. We will suppose also that $|S|=1 / 2$ as for true electrons. The Hamilton equations for the evolution of coordinates $(x, y)$ and momentum $\left(k_{x}, k_{y}\right)$ and LandauLifshitz equations for the evolution of spin components read

$$
\begin{gathered}
\dot{x}=\frac{k_{x}}{m}-2 \lambda S_{y}, \\
\dot{y}=\frac{k_{y}}{m}+2 \lambda S_{x}, \\
\dot{k}_{x}(t)=e E_{x}, \\
\dot{k}_{y}(t)=0, \\
\dot{\mathbf{S}}=-\Delta \times \mathbf{S},
\end{gathered}
$$

where $\boldsymbol{\Delta}$ is the effective magnetic field acting on the spin. From the Hamiltonian (A1) we can read this effective magnetic field acting on the particle with the momentum $\mathbf{k}$ $=\left(k_{x}, k_{y}\right)$,

$$
\boldsymbol{\Delta}=-2\left(\lambda k_{y},-\lambda k_{x}, h\right)
$$

The absolute magnitude of this field is

$$
|\boldsymbol{\Delta}|=\sqrt{4\left(k^{2} \lambda^{2}+h^{2}\right)} .
$$

Assuming that the electric field $E_{x}$ is weak, the variation of the momentum and hence of the effective magnetic field is very slow and, if at the initial moment the classical spin is directed along the magnetic field, it will follow the direction of this slowly varying field. This adiabatic approximation follows from the Landau-Lifshitz equations and yields an approximate solution for Eq. (A6) given by $\mathbf{S}^{(a)}(t)$ $= \pm|S|(\boldsymbol{\Delta}(t) /|\boldsymbol{\Delta}|)$, where "+" and "-" correspond to the initial direction along or opposite to the magnetic field, respectively. Substituting this time dependence of the spin direction into equations for particle velocities one can find that in the adiabatic approximation

$$
\begin{gathered}
\dot{x}=\frac{\partial \epsilon_{0}(\mathbf{k})}{\partial k_{x}}, \\
\dot{y}=\frac{\partial \epsilon_{0}(\mathbf{k})}{\partial k_{y}},
\end{gathered}
$$

where $\epsilon_{0}(\mathbf{k})=k^{2} / 2 m \mp \sqrt{(k \lambda)^{2}+h^{2}}$, i.e., a particle with spin along the field $\Delta$ has energy dispersion as an electron in the major band of R2DEG. In the strict adiabatic approximation of this classical Hamiltonian particles do not exhibit the anomalous velocity seen in the second term of the group velocity of the quantum-mechanical wave-packet dynamics described by Eq. (2).

However, this classical model does capture the correct contribution to the anomalous Hall effect related to the Berry curvature once we go beyond the strict adiabatic approximation and consider instead the more general linear response. The nonadiabatic corrections describe deflection of the classical spin from the direction of the instant magnetic field. They are small but can still be of the first order in the electric field and hence affect the transport properties in the linear response. Looking for a solution of the Landau-Lifshitz equations (A6) in the form $\mathbf{S}=\mathbf{S}^{(a)}+\delta \mathbf{S}$ we find that in the first order in the external electric field the spin direction acquires the component perpendicular both to the effective field and its derivative, ${ }^{25}$ i.e.,

$$
\delta \mathbf{S}= \pm|S| \frac{\boldsymbol{\Delta}}{|\boldsymbol{\Delta}|^{3}} \times \frac{d \boldsymbol{\Delta}}{d t} .
$$

If $h \neq 0$ this correction has in-plane components and hence affects the velocity of the particle, i.e., it gives a contribution to the right-hand side of Eqs. (A2) and (A3). Substituting Eqs. (A7) and (A8) into Eq. (A10) we find

$$
\begin{aligned}
& \delta S_{x}(\mathbf{k})= \pm|S| \frac{\lambda h \dot{k}_{x}}{2\left[(k \lambda)^{2}+h^{2}\right]^{3 / 2}}, \\
& \delta S_{y}(\mathbf{k})=\mp|S| \frac{\lambda h \dot{k}_{y}}{2\left[(k \lambda)^{2}+h^{2}\right]^{3 / 2}} .
\end{aligned}
$$

Then substituting Eqs. (A11) and (A12) into Eqs. (A2) and (A3) for $|S|=1 / 2$ we find equations identical to the wavepacket ones with the Berry curvature given in Eqs. (2) and (34).

We emphasize that although being an interesting demonstration of the effect, the model of classical charged particles with classical spins cannot always be employed in calculations of the Berry curvature and quantum corrections for other types of the Hamiltonian may appear. ${ }^{34}$
${ }^{1}$ R. Karplus and J. M. Luttinger, Phys. Rev. 95, 1154 (1954).

${ }^{2}$ F. Goos and H. Hänchen, Ann. Phys. (Leipzig) 1, 333 (1947);F. I.

Fedorov, Dokl. Akad. Nauk SSSR 105, 465 (1955).

${ }^{3}$ M. Onoda, S. Murakami, and N. Nagaosa, Phys. Rev. Lett. 93,
083901 (2004).

${ }^{4}$ K. Yu. Bliokh and V. D. Freilikher, cond-mat/0405384, Phys. Rev. B (to be published).

${ }^{5}$ T. Jungwirth, Q. Niu, and A. H. MacDonald, Phys. Rev. Lett. 88, 
207208 (2002).

${ }^{6}$ G. Sundaram and Q. Niu, Phys. Rev. B 59, 14915 (1999).

${ }^{7}$ V. S. Liberman and B. Ya. Zel'dovich, Phys. Rev. A 46, 5199 (1992).

${ }^{8}$ J. M. Luttinger, Phys. Rev. 112, 739 (1958).

${ }^{9}$ W. Kohn and J. M. Luttinger, Phys. Rev. 108, 590 (1957).

${ }^{10}$ J. Smit, Physica (Amsterdam) 21, 877 (1955); 24, 39 (1958).

${ }^{11}$ L. Berger, Phys. Rev. B 17, 1453 (1978).

${ }^{12}$ L. Berger, Phys. Rev. B 8, 2351 (1973).

${ }^{13}$ L. Berger, Phys. Rev. B 2, 4559 (1970).

${ }^{14}$ A. Crépieux and P. Bruno, Phys. Rev. B 64, 014416 (2001).

${ }^{15}$ V. K. Dugaev, A. Crépieux, and P. Bruno, Phys. Rev. B 64, 104411 (2001).

${ }^{16}$ S. K. Lyo and T. Holstein, Phys. Rev. Lett. 29, 423 (1972).

${ }^{17}$ Z. Fang, N. Nagaosa, K. S. Takahashi, A. Asamitsu, R. Mathieu, T. Ogasawara, H. Yamada, M. Kawasaki, Y. Tokura, and K. Terakura, Science, 302N5642, 92 (2003).

${ }^{18}$ S. H. Chun, M. B. Salamon, Y. Lyanda-Geller, P. M. Goldbart, and P. D. Han, Phys. Rev. Lett. 84, 757 (2000).

${ }^{19}$ Y. Yao, L. Kleinman, A. H. MacDonald, J. Sinova, T. Jungwirth, D. S. Wang, E. Wang, and Q. Niu, Phys. Rev. Lett. 92, 037204 (2004).

${ }^{20}$ E. N. Adams and E. I. Blount, J. Phys. Chem. Solids 10, 286 (1959).

${ }^{21}$ P. Nozieres and C. Lewiner, J. Phys. (France) 34, 901 (1973).

${ }^{22}$ J.-N. Chazalviel, Phys. Rev. B 11, 3918 (1975).
${ }^{23}$ G. H. Wannier, Phys. Rev. 117, 432 (1960).

${ }^{24}$ M. G. Vavilov and I. L. Aleiner, Phys. Rev. B 69, 035303 (2004).

${ }^{25}$ J. Sinova, D. Culcer, Q. Niu, N. A. Sinitsyn, T. Jungwirth, and A. H. MacDonald, Phys. Rev. Lett. 92, 126603 (2004); Shuichi Murakami, Naoto Nagaosa, and Shou-Cheng Zhang, Science 301, 1348 (2003).

${ }^{26}$ J. I. Inoue, G. E. W. Bauer, and L. W. Molenkamp, Phys. Rev. B 70, 041303 (2004).

${ }^{27}$ K. Nomura, J. Sinova, T. Jungwirth, Q. Niu, and A. H. MacDonald, Phys. Rev. B 71, 041304 (2005).

${ }^{28}$ E. G. Mishchenko, A. V. Shytov, and B. I. Halperin, Phys. Rev. Lett. 93, 226602 (2004).

${ }^{29}$ Compare, for example, with Fig. 1 in O. Costa de Beauregard, C. Imbert, and Y. Levy, Phys. Rev. D 15, 3553 (1977).

${ }^{30}$ D. Culcer, A. MacDonald, and Q. Niu, Phys. Rev. B 68, 045327 (2003).

${ }^{31}$ D. Culcer, J. Sinova, N. A. Sinitsyn, T. Jungwirth, A. H. MacDonald, and Q. Niu, Phys. Rev. Lett. 93, 046602 (2004).

${ }^{32}$ F. D. M. Haldane, Phys. Rev. Lett. 93, 206602 (2004).

${ }^{33}$ P. Bruno, V. K. Dugaev, and M. Taillefumier, Phys. Rev. Lett. 93, 096806 (2004).

${ }^{34}$ S. Murakami, N. Nagaosa, and S.-C. Zhang, Phys. Rev. B 69, 235206 (2004).

${ }^{35}$ V. K. Dugaev, P. Bruno, M. Taillefumier, B. Canals, and C. Lacroix, cond-mat/0502386. 